\title{
Compensation mechanism in silicon-doped gallium arsenide nanowires
}

\author{
B. Ketterer, ${ }^{1}$ E. Mikheev, ${ }^{1}$ E. Uccelli, ${ }^{1,2}$ and A. Fontcuberta i Morral ${ }^{1,2, a)}$ \\ ${ }^{1}$ Laboratoire des Matériaux Semiconducteurs, Institut des Matériaux, Ecole Polytechnique Fédérale de \\ Lausanne, 1015 Lausanne, Switzerland \\ ${ }^{2}$ Walter Scottky Institut und Physik Department, Technische Universität München, 85748 Garching, \\ Germany
}

(Received 4 September 2010; accepted 26 October 2010; published online 30 November 2010)

\begin{abstract}
P-type gallium arsenide nanowires were grown with different silicon doping concentrations. The incorporation is monitored by Raman spectroscopy of the local vibrational modes. For Si-concentrations up to $1.4 \times 10^{18} \mathrm{~cm}^{-3}$, silicon incorporates mainly in arsenic sites. For higher concentrations, we observe the formation of silicon pairs. This is related to the Coulomb interaction between charged defects during growth. An electrical deactivation of more than $85 \%$ of the silicon acceptors is deduced for nominal silicon concentration of $4 \times 10^{19} \mathrm{~cm}^{-3}$. This work is important to understand the limiting mechanisms of doping in compound semiconductor nanowires. () 2010 American Institute of Physics. [doi:10.1063/1.3517254]
\end{abstract}

Semiconductor nanowires are potential building blocks of multiple applications in diverse areas such as electronics, energy harvesting, and biosensing. ${ }^{1-5}$ For devices to become a technological reality, controlled and reproducible doping is essential. In the past few years, there have been studies focusing on the mechanisms and the limitations of dopant incorporation in nanowires. ${ }^{6-10}$ Various challenges have been anticipated and/or found, such as the competition of dopant incorporation between the shell and the core of the nanowire and doping compensation. ${ }^{6,7}$ Compensation can typically occur in amphoteric dopants such as silicon in GaAs. Indeed, $\mathrm{Si}$ can lead to n- or p-type doping, depending on whether it is incorporated in the Ga or As sites of the host lattice. Additionally, a correlation between the dopants incorporation can exist, in a way that the simple neutral complexes lay in nearest-neighbor position, forming donor acceptor pairs $\mathrm{Si}_{\mathrm{Ga}}-\mathrm{Si}_{\mathrm{As}}$. At extremely high doping concentrations, supersaturation and dopant precipitation can occur. ${ }^{11} \mathrm{Si}$ is most commonly used as n-type dopant in molecular beam epitaxy (MBE) grown (100) oriented GaAs thin films leading to carrier concentrations higher than $5 \times 10^{18} \mathrm{~cm}^{-3}$, with a majority of $\mathrm{Si}$ atoms being incorporated as $\mathrm{Si}_{\mathrm{Ga}}$ donors. ${ }^{12} \mathrm{In}$ contrast, n-type (110) films with the same silicon concentration show 30\%-40\% lower free-electron concentrations than their (100) counterparts. ${ }^{13}$ By lowering the $\mathrm{As}_{4} / \mathrm{Ga}$ flux ratio and increasing the growth temperature, $\mathrm{Si}$ incorporates preferentially in the As sites, giving rise to p-type doping. ${ }^{13-15} \mathrm{Re}-$ cently, silicon has been used for doping GaAs nanowires and both $\mathrm{n}$ - and $\mathrm{p}$-type doping have been achieved. The type of doping seems to depend on the growth temperature. Indeed, at $570{ }^{\circ} \mathrm{C}$ silicon tends to incorporate as a donor, while at $630{ }^{\circ} \mathrm{C}$ it is incorporated as an acceptor. ${ }^{6,16}$ The dopant incorporation can be monitored by measuring the local vibrational modes (LVMs) associated with the impurities. One advantage of Raman spectroscopy over electrical measurements is that spurious effects coming from the fabrication of contacts can be avoided and information on the type and concentration of dopants is easily obtained. ${ }^{17}$

In a previous study, we have elucidated the silicon doping mechanisms of catalyst-free GaAs nanowires. We have

\footnotetext{
a) Author to whom correspondence should be addressed. Electronic mail: anna.fontcuberta-morral@epfl.ch.
}

shown that silicon incorporates as an acceptor both on the facets and the nanowire core. A schematic drawing of the final structure is shown in Fig. 1(a). This leads to the formation of a highly p-doped shell on the nanowire core, a consequence of the nonzero radial growth of the nanowire. In
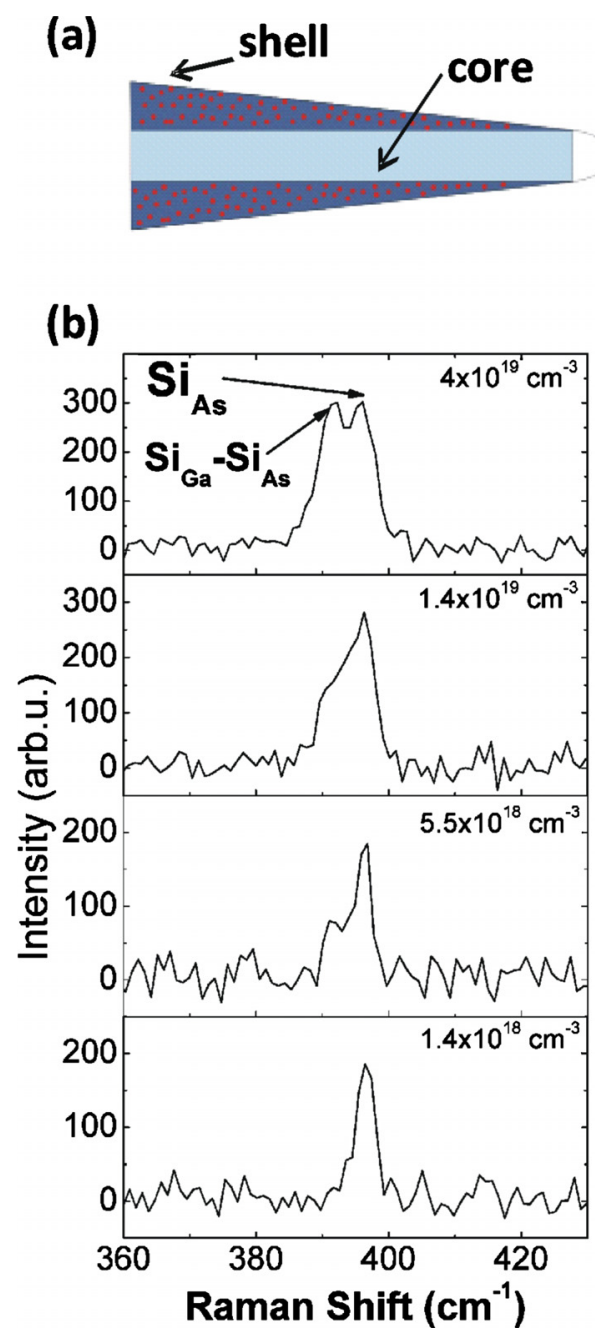

FIG. 1. (Color online) (a) Schematic drawing of the incorporation of silicon in the nanowire: on the side facets through radial growth and in the core through the droplet. (b) Raman spectra of the Si-doped GaAs nanowires for different Si concentrations in the shell. 
this work, we demonstrate the existence of compensation in the shell and find the corresponding mechanisms.

Catalyst-free p-type GaAs nanowires were prepared by MBE in a Gen II system as described elsewhere. ${ }^{6,18}$ The substrate was rotated at $7 \mathrm{rpm}$ and heated at $630^{\circ} \mathrm{C}$. We used a nominal Ga growth rate of $0.25 \AA / \mathrm{s}$, an $\mathrm{As}_{4}$ partial pressure of $2.7 \times 10^{-6}$ mbar. The growth time was $4 \mathrm{~h}$, resulting in $22 \mu \mathrm{m}$ long tapered wires with a diameter ranging between 180 and $70-90 \mathrm{~nm}$ (from the bottom to the tip). ${ }^{6}$ The tapering is a consequence of a diameter growth rate of $0.008 \mathrm{~nm} / \mathrm{s}$. Silicon doping was achieved by adding a silicon flux during the nanowire growth. Samples were prepared with a silicon flux of $5.6 \times 10^{9}, 2.2 \times 10^{10}, 5.6 \times 10^{10}$, and $1.6 \times 10^{11}$ at $\mathrm{Si} /\left(\mathrm{cm}^{2} \mathrm{~s}\right)$. Taking into account the nanowire geometry and radial growth rate, this corresponds to a silicon concentration in the shell of $1.4 \times 10^{18}, 5.5 \times 10^{18}, 1.4$ $\times 10^{19}$, and $4 \times 10^{19} \mathrm{~cm}^{-3}$.

The structure of the nanowires corresponds to $100 \%$ zinc-blende with the occurrence of single twins along the nanowire axis. ${ }^{6,19,20}$ Raman spectra were recorded in backscattering geometry at $90 \mathrm{~K}$. The $488 \mathrm{~nm}$ line of an $\mathrm{Ar}^{+} \mathrm{Kr}^{+}$ laser was used for excitation. The laser was focused on the nanowire with a cover glass corrected objective (0.75 NA) (NA denotes numerical aperture) with the incident polarization parallel to the nanowire axis. A xyz-piezostage with a precision of $<2 \mathrm{~nm}$ allowed the scanning of the laser spot along the axis of a single nanowire dispersed on a Si substrate. The power of the incident light was $500 \mu \mathrm{W}$. The scattered light was collected by a TriVista triple spectrometer with a multichannel cooled charge-coupled-device detector.

Typical Raman spectra of the GaAs nanowires grown with the various silicon fluxes are shown in Fig. 1(b). All the measurements have been taken with the laser spot positioned at a distance of $1 \mu \mathrm{m}$ from the base. At that point, the doped shell is about $60 \mathrm{~nm}$ thick. As the Raman information depth at $488 \mathrm{~nm}$ is about $40 \mathrm{~nm}$, the Raman spectra report only about the doping mechanisms in the nanowire shell and not from the core. In all Raman spectra, we observe a $\mathrm{Si}_{\mathrm{As}} \mathrm{LVM}$ at $396 \mathrm{~cm}^{-1}$ that can be attributed to the incorporation of $\mathrm{Si}$ into As lattice sites. This mode is a characteristic for the p-type doping. ${ }^{21}$ For nanowires grown with a Si-flux larger than $5.6 \times 10^{9} \mathrm{Si} /\left(\mathrm{cm}^{2} \mathrm{~s}\right)$, an additional mode arises at $393 \mathrm{~cm}^{-1}$ that can be assigned to the formation of neutral $\mathrm{Si}_{\mathrm{Ga}}-\mathrm{Si}_{\mathrm{As}}$ pairs. ${ }^{12}$ The intensity of this peak increases as the silicon flux is increased. For the nanowires obtained with the maximum silicon flux, the two peaks associated with the sites $\mathrm{Si}_{\mathrm{As}}$ and $\mathrm{Si}_{\mathrm{Ga}}-\mathrm{Si}_{\mathrm{As}}$ have a similar intensity.

For a given scattering volume and scattering cross section per impurity, the measured LVM intensity is directly proportional to the impurity concentration. ${ }^{22}$ Thus, Raman scattering allows the relative quantitative characterization of the dopant incorporation. In Fig. 2(a), the integrated intensity of the $\mathrm{Si}_{\mathrm{As}} \mathrm{LVM}$ is plotted versus the incident Si-flux during the growth. For a fair comparison of the data, the intensity of the $\mathrm{Si}_{\mathrm{As}} \mathrm{LVM}$ has been normalized to the intensity of the transverse optical GaAs mode, whose intensity is proportional to the volume probed. Interestingly, we find a sublinear increase of the $\mathrm{Si}_{\mathrm{As}} \mathrm{LVM}$ intensity with respect to the total incoming Si-flux. This behavior is a signature of the existence of dopant compensation at higher doping levels. Now, we compare the increase in the incorporation of $\mathrm{Si}$ in As sites and the formation of Si-pairs. The intensity ratio
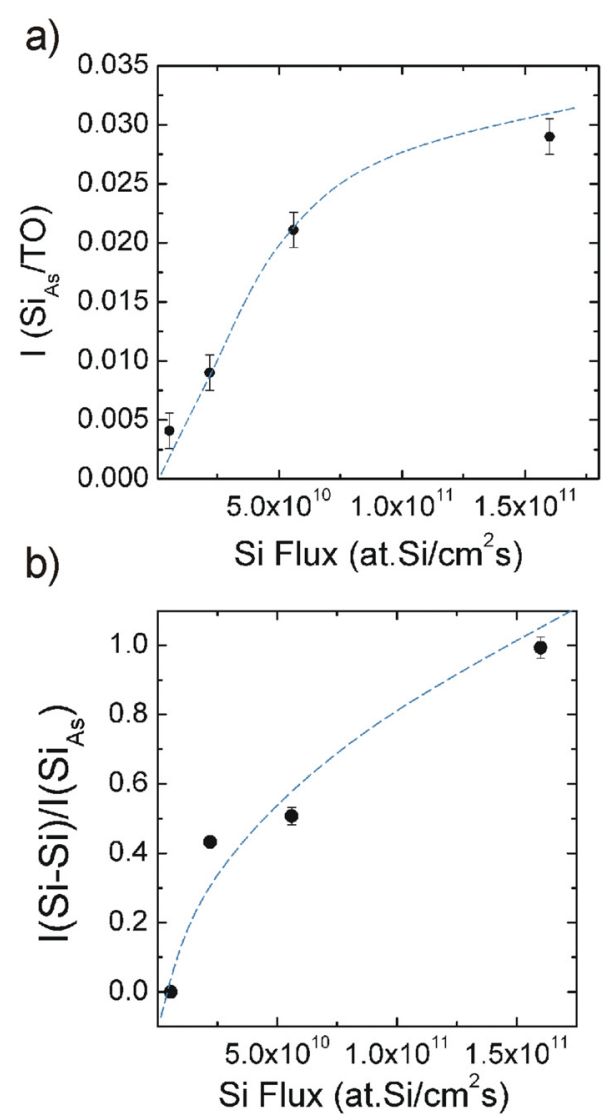

FIG. 2. (Color online) (a) Integrated intensity of the normalized $\mathrm{Si}_{\mathrm{As}}$ mode as a function of the incident Si-flux. (b) Intensity ratio between the $\mathrm{Si}_{\mathrm{Ga}}-\mathrm{Si}_{\mathrm{As}}$ and $\mathrm{Si}_{\mathrm{As}}$ LVMs as a function of the Si-flux.

between the LVM of $\mathrm{Si}_{\mathrm{As}}-\mathrm{Si}_{\mathrm{Ga}}$ and $\mathrm{Si}_{\mathrm{As}}$ as a function of the silicon flux is shown in Fig. 2(b). In this case, the increase of the mode related to the silicon pairs is quite abrupt and tends to saturate at the highest doping. This is a signature that the compensation occurs in a nonrandom way.

The main source of compensation in p-type GaAs: $\mathrm{Si}$ is $\mathrm{Si}$ atoms incorporated in $\mathrm{Ga}$ lattice sites $\left(\mathrm{Si}_{\mathrm{Ga}}\right)$ acting as donors. Interestingly, it has been found that the reduced screening of the Coulomb interaction between the charged defects can be the driving mechanism for the formation of neutral Si-pairs. ${ }^{23}$ As a consequence of the consecutive compensation of $\mathrm{Si}_{\mathrm{As}}$ acceptors by $\mathrm{Si}_{\mathrm{Ga}}$ donors, the effective free carrier concentration decreases. Thus, the efficiency of the screening of the electric fields originating from charged atoms is reduced. When the average distance between two $\mathrm{Si}$ atoms in the host lattice becomes similar or smaller than the screening length, the Coulomb interaction between the negatively charged $\mathrm{Si}$ acceptors and positively charged $\mathrm{Si}$ donors leads to the formation of neutral Si-pairs. Such a pair formation occurs during the growth process. For a nondegenerate semiconductor, the characteristic length scale for Coulomb interaction is given by the Debye screening length $\lambda_{\mathrm{D}}$,

$$
\lambda_{D}=\left(\frac{\varepsilon_{0} \cdot \varepsilon \cdot k_{B} \cdot T}{e^{2} \cdot p^{*}}\right)^{1 / 2},
$$

where $\varepsilon=13.1$ is the permittivity of GaAs and $p^{*}$ is the effective screening density of free carriers. The Debye length as a function of the effective carrier concentration in GaAs at $630{ }^{\circ} \mathrm{C}$ is displayed in Fig. 3. In the same graph, we plot the average $\mathrm{Si}-\mathrm{Si}$ distance for the concentrations of the nano- 


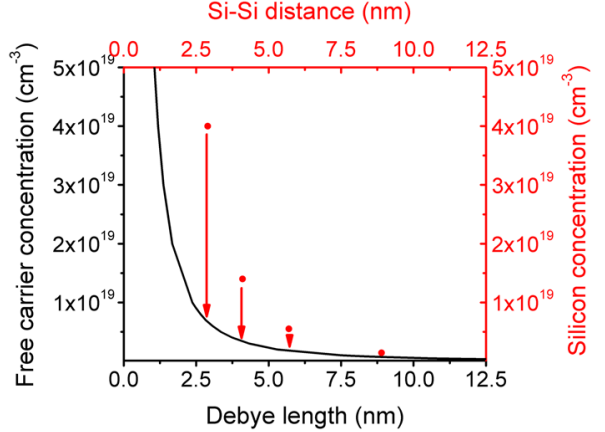

FIG. 3. (Color online) Calculated Debye screening length as a function of the effective free carrier concentration (full line) and the average $\mathrm{Si}-\mathrm{Si}$ distance for the total Si concentrations of $1.4 \times 10^{18}, 5.5 \times 10^{18}, 1.4 \times 10^{19}$, and $4 \times 10^{19} \mathrm{~cm}^{-3}$. The arrows indicate the minimum carrier concentration for each of the silicon concentrations.

wires presented in this work, in the case where the distribution of dopants is completely random. By comparing the average $\mathrm{Si}-\mathrm{Si}$ distance with $\boldsymbol{\lambda}_{\boldsymbol{D}}$ for the corresponding free carrier concentration, it is possible to deduce if Coulomb interaction between charged defects can drive the formation of Si-pairs. Additionally, it is possible to deduce the lower bound of compensation.

The average distance between two silicon atoms supposing a random distribution of the dopants for silicon concentrations of $4 \times 10^{19}, 1.4 \times 10^{19}, 5.5 \times 10^{18}$, and 1.4 $\times 10^{18} \mathrm{~cm}^{-3}$ is respectively $2.9,4.1,5.7$, and $8.9 \mathrm{~nm}$. Now, looking back at the experimental data in Fig. 1, it is apparent that a certain threshold value for the Si-pair formation exists that is overstepped when the total $\mathrm{Si}$-concentration is increased from $1.4 \times 10^{18}$ to $5.5 \times 10^{18} \mathrm{~cm}^{-3}$. At this threshold concentration, the corresponding $\mathrm{Si}-\mathrm{Si}$ distance crosses over from a value that is higher than the Debye length to a value lower than $\lambda_{\mathrm{D}}$.

For a Si-concentration below the threshold, as for the lowest Si-concentration of $1.4 \times 10^{18} \mathrm{~cm}^{-3}$, there is no experimental evidence for $\mathrm{Si}-\mathrm{Si}$ pair formation. Consequently, $\lambda_{\mathrm{D}}$ has to be smaller than the average $\mathrm{Si}-\mathrm{Si}$ distance of 8.9 $\mathrm{nm}$. As we can see in Fig. 3, the difference between the total Si-concentration and the effective free carrier concentration corresponding to this upper bound of $\lambda_{\mathrm{D}}=8.9 \mathrm{~nm}$ is negligible. Therefore, in excellent accordance with the model of screened Coulomb interaction, compensation effects can be neglected for nanowires grown with the lowest $\mathrm{Si}$-flux. At the same time, we can give an estimate for a free carrier concentration of $1.4 \times 10^{18} \mathrm{~cm}^{-3}$ in this sample (the contribution of the intrinsic carrier concentration to the total free carrier concentration in GaAs is still negligible at the growth temperature of $903 \mathrm{~K}$ ).

On the other hand, for Si concentrations above the critical threshold value, we know from the experimental observation of Si-pairs that the screening length is effectively larger than the Si-Si distance. For example, for the sample grown with the highest Si-concentration of $4 \times 10^{19} \mathrm{~cm}^{-3}$, the Debye length has to be larger than the $\mathrm{Si}-\mathrm{Si}$ distance of $2.9 \mathrm{~nm}$. Figure 3 shows that for $\lambda_{\mathrm{D}}>2.9 \mathrm{~nm}$, the effective free carrier concentration is smaller than $6 \times 10^{18} \mathrm{~cm}^{-3}$. Therefore, the difference between the total Si-concentration and the effective free carrier concentration is at least 3.4 $\times 10^{19} \mathrm{~cm}^{-3}$ (this difference is denoted by an arrow in Fig. 3 ), corresponding to a minimum compensation of $85 \%$ in the nanowires grown with the highest $\mathrm{Si}$-flux. This means that at least $85 \%$ of the $\mathrm{Si}$ atoms do not contribute to the carrier concentration. Finally, from these considerations, we can also give an upper bound of the free carrier concentration of $6 \times 10^{18} \mathrm{~cm}^{-3}$ for the nanowire grown with the highest $\mathrm{Si}$ flux. These findings are in good agreement with our previous results. ${ }^{6}$

In summary, the effect of dopant compensation in Sidoped p-type GaAs nanowires has been investigated by Raman spectroscopy. We have shown that the compensation results in a sublinear increase in the $\mathrm{Si}_{\mathrm{As}}$ concentration with the incoming Si-flux. The reduced Coulomb screening drives the formation of Si-pairs and results in highly compensated GaAs for a silicon concentration higher than 1.4 $\times 10^{18} \mathrm{~cm}^{-3}$. An observed electrical deactivation of at least $85 \%$ of the $\mathrm{Si}$ acceptors is observed for nominal silicon concentration of $4 \times 10^{19} \mathrm{~cm}^{-3}$. These results will have to be taken into consideration in the future for the fabrication of optical and electronic devices with Si-doped GaAs nanowires.

The authors would like to kindly thank G. Abstreiter and M. Bichler for their experimental support. This research was supported by the SNF Grant No. 2000021/12175 the ERC Starting grant "Upcon," and the DFG excellence cluster Nanosystems Initiative Munich.

${ }^{1}$ W. U. Wang, C. Chen, K. H. Lin, Y. Fang, and C. M. Lieber, Proc. Natl. Acad. Sci. U.S.A. 102, 3208 (2005).

${ }^{2}$ G. F. Zheng, F. Patolsky, Y. Cui, W. U. Wang, and C. M. Lieber, Nat. Biotechnol. 23, 1294 (2005).

${ }^{3}$ Y. Cui and C. M. Lieber, Science 291, 851 (2001).

${ }^{4}$ S. J. Tans, A. R. M. Verschueren, and C. Dekker, Nature (London) 393, 49 (1998).

${ }^{5}$ B. M. Kayes, H. A. Atwater, and N. S. Lewis, J. Appl. Phys. 97, 114302 (2005).

${ }^{6}$ J. Dufouleur, C. Colombo, T. Garma, B. Ketterer, E. Uccelli, M. Nicotra, and A. Fontcuberta i Morral, Nano Lett. 10, 1734 (2010).

${ }^{7}$ D. E. Perea, E. R. Hemesath, E. J. Schwalbach, J. L. Lensch-Falk, P. W. Voorhees, and L. J. Lauhon, Nat. Nanotechnol. 4, 315 (2009).

${ }^{8}$ E. C. Garnett, Y.-C. Tseng, D. R. Khanal, J. Wu, J. Bokor, and P. Yang, Nat. Nanotechnol. 4, 311 (2009).

${ }^{9}$ P. V. Radovanovic, C. J. Barrelet, S. Gradecak, F. Qian, and C. M. Lieber, Nano Lett. 5, 1407 (2005).

${ }^{10}$ C. Gutsche, I. Regolin, K. Blekker, A. Lysov, W. Prost, and F. J. Tegude, J. Appl. Phys. 105, 024305 (2009).

${ }^{11}$ D. T. J. Hurle, J. Appl. Phys. 85, 6957 (1999).

${ }^{12}$ R. C. Newman, Mater. Sci. Eng., B 66, 39 (1999).

${ }^{13}$ J. M. Ballingall and C. E. C. Wood, Appl. Phys. Lett. 41, 947 (1982).

${ }^{14}$ E. S. Tok, J. H. Neave, M. J. Ashwin, B. A. Joyce, and T. S. Jones, J. Appl. Phys. 83, 4160 (1998).

${ }^{15}$ F. Fischer, D. Schuh, M. Bichler, G. Abstreiter, M. Grayson, and K. Neumaier, Appl. Phys. Lett. 86, 192106 (2005).

${ }^{16}$ M. Hilse, M. Ramsteiner, S. Breuer, L. Geelhaar, and H. Riechert, Appl. Phys. Lett. 96, 193104 (2010).

${ }^{17}$ B. Prévot and J. Wagner, Prog. Cryst. Growth Charact. Mater. 22, 245 (1991).

${ }^{18}$ C. Colombo, D. Spirkoska, M. Frimmer, G. Abstreiter, and A. Fontcuberta i Morral, Phys. Rev. B 77, 155326 (2008).

${ }^{19}$ D. Spirkoska, J. Arbiol, A. Gustafsson, S. Conesa-Boj, I. Zardo, M. Heigoldt, M. H. Gass, A. L. Bleloch, S. Estrade, F. Peiro, J. R. Morante, G. Abstreiter, L. Samuelson, and A. Fontcuberta i Morral, Phys. Rev. B 80, 245325 (2009).

${ }^{20}$ I. Zardo, S. Conesa-Boj, F. Peiro, J. R. Morante, J. Arbiol, E. Uccelli, G. Abstreiter, and A. Fontcuberta i Morral, Phys. Rev. B 80, 245324 (2009)

${ }^{21}$ M. J. Ashwin, M. R. Fahy, R. C. Newman, J. Wagner, D. A. Robbie, M. J. L. Sangster, I. Silier, E. Bauser, W. Braun, and K. Ploog, J. Appl. Phys. 76, 7839 (1994).

${ }^{22}$ K. Jain, S. Lai, and M. V. Klein, Phys. Rev. B 13, 5448 (1976).

${ }^{23}$ C. Domke, Ph. Ebert, M. Heinrich, and K. Urban, Phys. Rev. B 54, 10288 (1996). 